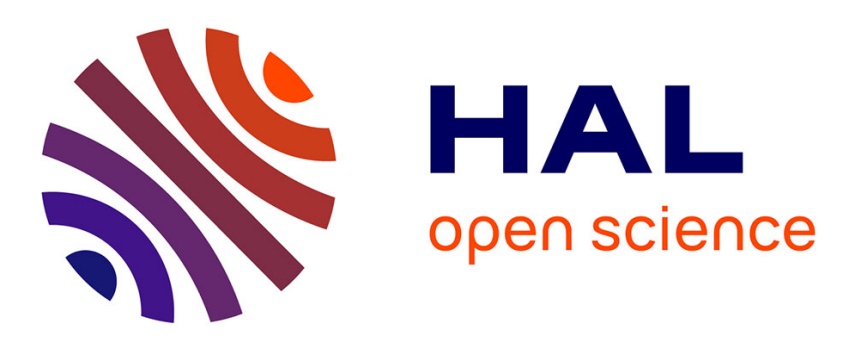

\title{
Everyday Creative Uses of Smartphone Images in Biomedical Engineering Laboratories
}

\author{
Dhaval Vyas, Hinal Vyas, Maria A. Woodruff
}

\section{To cite this version:}

Dhaval Vyas, Hinal Vyas, Maria A. Woodruff. Everyday Creative Uses of Smartphone Images in Biomedical Engineering Laboratories. 16th IFIP Conference on Human-Computer Interaction (INTERACT), Sep 2017, Bombay, India. pp.335-343, 10.1007/978-3-319-67744-6_22 . hal-01676174

\section{HAL Id: hal-01676174 \\ https://hal.inria.fr/hal-01676174}

Submitted on 5 Jan 2018

HAL is a multi-disciplinary open access archive for the deposit and dissemination of scientific research documents, whether they are published or not. The documents may come from teaching and research institutions in France or abroad, or from public or private research centers.
L'archive ouverte pluridisciplinaire HAL, est destinée au dépôt et à la diffusion de documents scientifiques de niveau recherche, publiés ou non, émanant des établissements d'enseignement et de recherche français ou étrangers, des laboratoires publics ou privés. 


\title{
Everyday Creative Uses of Smartphone Images in Biomedical Engineering Laboratories
}

\author{
Dhaval Vyas ${ }^{1}$, Hinal Vyas ${ }^{2}$, Maria A. Woodruff ${ }^{2}$ \\ Queensland University of Technology (QUT) \\ ${ }^{1}$ School of Electrical Engineering and Computer Science (EECS), \\ 2 George Street, Brisbane, QLD, 4000, Australia \\ ${ }^{2}$ Institute of Health and Biomedical Innovation (IHBI), \\ 60 Musk Avenue, Kelvin Grove, QLD 4059, Australia \\ \{d.vyas | h.vyas | mia.woodruff\}@qut.edu.au
}

\begin{abstract}
In this paper, we focus on creative practices associated with smartphone images for supporting scientific work. We employed observations and semi-structured interviews with 12 research staff members from a biomedical engineering institute over a period of three months and explored the role smartphone images play in supporting their scientific activities. We studied different ways smartphone images are incorporated into researchers' everyday work. Our findings highlight practices and motivations associated with the use of smartphone images. Based on our findings, we provide implications for designing innovative smartphone apps and particularly emphasize the role smartphones can play in developing and maintaining hybrid lab-books.
\end{abstract}

\section{Introduction}

Studies of science and technology $[28,12]$ have pointed to the fact that while final outcomes of any scientific work, be it scientific articles, functional technologies or newspaper reports, inform about scientific facts and truths, a large number of procedural insights and local contingency are often filtered out. In particular, how scientists come about making sense of their data, images, or other type of information and what cognitive processes manifest themselves is rarely reported. The conduct of scientific research involves a varied set of cognitive processes and skills. Some of these are internal processes of the sort that have been the focus of the traditional cognitive science for decades, such as, categorization, reasoning, problem solving, and analogy formation. Others are processes that take place when information is propagated across different representational media, such as documents, papers and other types of external representations (e.g. [10, 11, 26, 27, 30]). Researchers have recognized that cognition is a socially and culturally embedded phenomenon that is situated and distributed between people concerned [8, 10, 11, 17, 21, 26, 27]. Cognition is as much rooted in mental processes as it is in the external world of objects, artifacts and social practices. In particular, the importance of external representations in reasoning and knowledge 
construction has been noted by many researchers seeking to understand the nature of the science $[11,14,16,20]$.

The current generation of smartphone cameras with advanced capabilities for rapidly capturing and sharing images has shown a great potential for the development of innovative applications that can support medical and scientific practices. Smartphonebased applications such as CellScope [5], M-Health [2], smartCARD [23], and mobile spectroscopy [9] have shown how current smartphones can be augmented to support scientific processes. There are similar examples $[6,7]$ in the field of ophthalmology. While the development of such bespoke solutions is increasing, there is a lack of research into studying existing practices of smartphone use in scientific work. Human Computer Interaction (HCI) researchers can develop innovative solutions, when there is a strong repertoire of knowledge about the role smartphone images play in supporting scientific work.

In order to explore this area, we carried out an ethnographic study at a biomedical engineering facility in a university setting. We involved 12 researchers in a semistructured interview study and an observational study. We studied their everyday work practices and in particular observed their use of smartphone images. Using an inductive thematic analysis approach [3], we organized our findings in the form of social 'practices' and 'motivations' associated with the use of smartphones.

As a common laboratory protocol, all the researchers kept lab-books where they recorded each and every minor detail of their activities. A detailed analysis of these lab-book showed that capturing images using smartphones at different stages of their work was a very common practice. We found four generic types of smartphone images: microscopic images, procedural images, equipment images, and measurement images. We found that these images were used for recording complex information, supported coordination and communication within teams, worked as a referencing and troubleshooting tool and became a simple way of information offloading. Based on such practices we point to some important implications for designing new mobile solutions, in particular exploring the design of physical-digital lab-books.

We make two contributions to the HCI community by 1 ) developing an empirical understanding of the smartphone image use in the biomedical engineering research; and 2) providing important implications for designing novel mobile solutions.

\section{Methods}

We used two methods to understand the use of smartphone images in scientific work. We contacted three research labs within the biomedical engineering facility and recruited 12 participants for a semi-structured interview study. 8 out of these 12 participants agreed to let us observe their lab work. Table 1 provides details of these participants. The three labs that were approached were working in the areas of Biomaterial and Tissue, Bones and Histology, which combined a good variation of expertise and focus on biomedical engineering [18]. 
Table 1. Participants in interviews and observations.

\begin{tabular}{|c|c|c|}
\hline Laboratory & Participants & Methods \\
\hline Biomaterials \& Tissue & 5 & Interview (5), Observations (3) \\
\hline Bones & 4 & Interview (4), Observations (2) \\
\hline Histology & 3 & Interview (3), Observations (3) \\
\hline
\end{tabular}

Our participants included lab technicians, senior researchers and $\mathrm{PhD}$ students in their final years. All of them had at least 3 years of experience working in the field. In the interview sessions, we asked our participants to describe their laboratory work and processes, we looked though their lab-books and smartphones to understand the role of digital images. We also asked them to discuss at least three recent projects using their lab-books. The interviews lasted for about 45 minutes and were audio recorded and later transcribed. We took photos of their lab-book pages and smartphone images while they answered our questions. We also followed 8 of these participants in their laboratory work. We made scheduled appointments with these participants over three months. We spent nearly 30 hours with them observing their activities, and specifically looking into their use of smartphones during this time. We took notes while our visits to the laboratories and audio recorded conversations. We carried out thematic analysis [3] on the participants' observation and interview data, where we inductively identified patterns and themes within the data in the form of social practices and goals.

\section{Results I: Practices}

In this section, we will discuss specific practices related to the use of smartphone images.

\subsection{Use of lab-book}

All the researchers working in these labs were required to keep their lab-books up-todate. Smartphone cameras were used to capture several of the scientific and procedural details in the form of images and in rare cases videos. Our participants used their lab-books to record a large variety of information: images of their experimental activities, hand written notes, annotations and drawings, printed email exchanges and standard operating procedures (SOP), post-its, bookmarks, graphs of their results, among other things. This way, lab-books contained both physical and digital (later printed and glued to the pages) information. Affordances of the paper-based lab-book allowed participants to share among team members, photocopy them or add pages whenever required. These features are highlighted in the work of Sellen and Harper [27]. We observed that researchers used a large amount of digitally-created infor- 
mation (smartphone images, graphs, email prints) which was printed and glued their printed versions onto their physical lab-books.

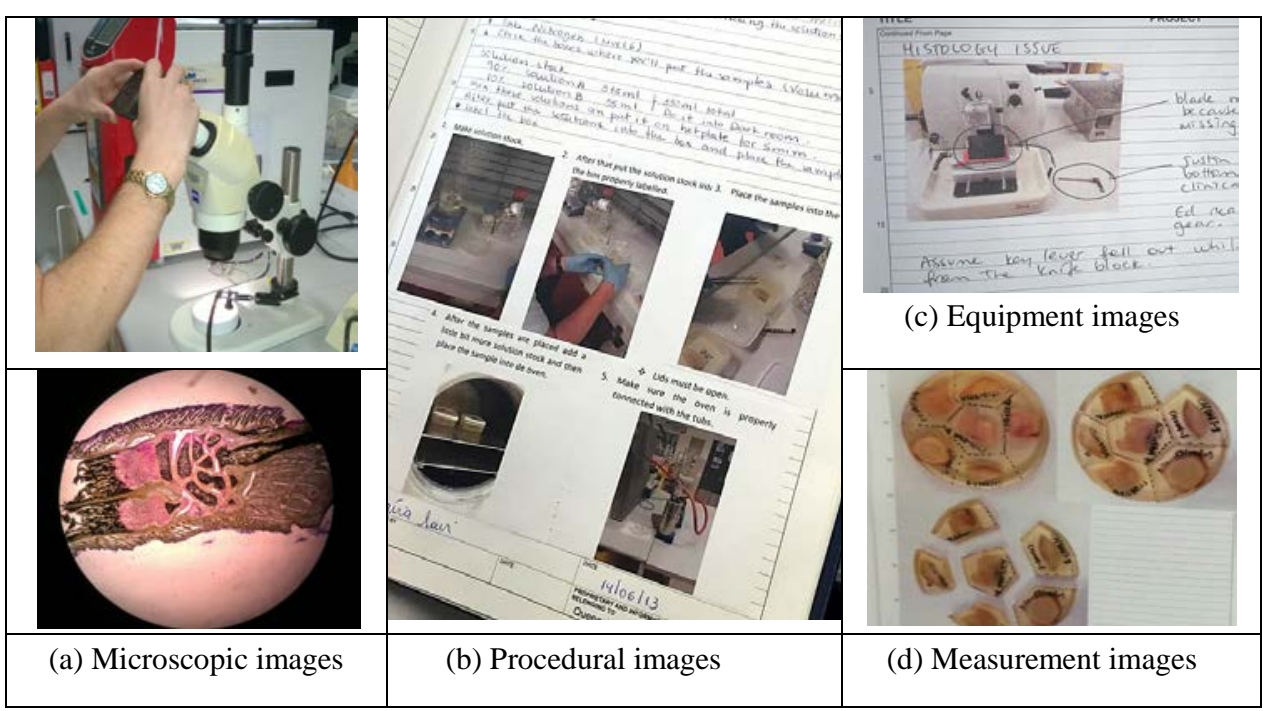

Figure 1. Images captured using smartphones.

\subsection{Types of images}

We observed that our participants captured images from their smartphones for different purposes. Figure 1 shows some examples that we collected during our research. We categorize them into four generic types:

1. Microscopic images are captured by placing smartphones directly onto the eye piece of microscopes (Figure 1a). These images are captured to check the morphology (e.g. shapes, colors, structures) of different biological samples. In the labs that we studied, some of the microscopes did not have the in-built camera feature and this was where such a use of smartphone cameras was useful. We found that participants captured such images because it was quicker to transfer, share and print images. Participants commented that such images are not used in important publications, but these provided good indications on the progress of their experiments.

2. Procedural Images are captured during the regular course of an experiment where the aim is to record each and every step through projects. Figure 1b shows a labbook page which captures all the steps taken in an experiment, allowing others to reproduce the process in future. Images captured in such a way also allow researchers to troubleshoot when they come across any problem.

3. Equipment Images are captured to report specific ways of using biomedical equipment or point out any issues (or workarounds) so that other lab workers can get informed. Figure 1c shows a lab-book page where an issue in an instrument is report- 
ed with annotated instructions. These types of images are captured to help others in the lab who might use those equipment.

4. Measurement Images are captured to record sample sizes, sample labels, chemical quantity, among other technical information that are important to specific experiments. These images serve as reminders and can be re-visited during the troubleshooting phase. Figure 1d shows an image where samples and their labels are captured.

\section{Results II: Motivations and goals}

In this part, we will discuss the motivations and goals behind using smartphone images in biomedical research.

\subsection{Quick recordkeeping, reliability and troubleshooting}

We observed that the use of smartphone images in lab-books served multiple uses. All of our participants maintained a very meticulous lab-book in order to make sure that they record each and every step of their work. During an interview session, one of the participants said: "There are quite a few different things which we need to record. Perhaps starting from the very beginning - the processing and embedding of tissue usually requires substantial amount of sequential and time-based steps so you need to record quite accurately what solution you are changing from and into; all that needs to be documented quit well. Mobile photos help in this case."

The role of smartphone images was quite important in the development of the labbooks. Smartphones being ready-at-hand allowed recording very complex set of information easily and quickly. In the case of the microscopic images (Figure 1a), researcher did not have to rely on the inbuilt camera of the microscope. During a typical experiment once samples are embedded with some chemicals, researchers needed to regularly check the behaviour of the sample on specific microscopes. We observed that researchers relied on their smartphone images because it was easier and quicker to do preliminary analysis on their samples. Images captured through the built-in cameras of microscope were high-res and hence quite large in size, which made the sharing, transferring and printing process slower for participants. It was a compromise in quality, but in cases where quick analysis is required (changes in cells colour or size), this compromise was not counterproductive for the research.

During experiments participants would have to prepare a large number of samples with different permutations and remembering them or even keeping a hand-written record of individual samples would be a tedious job. Figure 1d shows an example where a researcher has used images to keep track of their samples, reliably. The image highlights the issue of labelling and orientation. In this example a participant working on a sheep tibia bone had sectioned a large sample in different orientations. She has labelled these samples and captured images of these samples with their labels so that she can keep track of different procedures followed on these samples. She commented: "As we process our samples, we maintain a clear chain of orientation. 
We take lots of pictures of the samples: how they have been processed. It is little effort but we are always confident of what we are doing and we can always go back to our log-book images and check."

Several participants commented about the problems they faced in maintaining their lab-books. Some participants pointed out that the amount of time and effort spent on capturing images through smartphones and later attaching them on their lab-books was really high. Others commented that the issue of labeling (when the samples are in large numbers) was a big problem. Some participants commented about the resolution issues of smartphone images (in comparison to the high definition cameras built into certain biomedical equipment). These are the clear points where technology can play a role and novel solutions can be explored.

\subsection{Coordination and communication}

Smartphone images allowed smoother coordination and communications within and outside of the teams. Often the work was handed over to other members within the same lab and having all the details captured in lab-books helped in coordinating activities. The facility had collaborations with several international universities and medical centers. They would get samples from these collaborators to carry out specialized experiments. A participant commented: "We have to make sure that our collaborators know what we are planning to do with their samples. We show them images of how we sectioned their samples before we do anything on it..... Since I keep my lab book up to date, my team members can also take over if I am not available."

Even within the facility, there were specific cases where smartphone images helped in communicating difficult issues with other members. One of the participants who participated in our study commented the following: "I was doing this work for one of the PhD students in our group. I wanted to show him that this tissue was hard and without making it soft enough I could not section it properly. At that time, I showed him some photos and discussed this issue and after the discussion we decided how to work on this particular section."

\section{Discussion and implications}

Our interaction with three research laboratories highlights how the use of smartphone images has become interweaved with the everyday work practices of the researchers. In several cases, the use of smartphone images was simply a quick workaround. In the case of microscopic images (Figure 1a), the ready-at-hand nature of smartphones allowed researchers to take photos directly through the eye piece of microscopes and to have quick prints. This particular workaround had some issues with the quality of images. These images also played a role of cognitive offloading, in a sense that rather than having to remember the measures or names of chemicals, capturing an image using smartphones would be very easy to make sure that the precise information is recorded. Another issue that our research has highlighted is the empirical aspect of meticulous recording of information. Even when researchers had to go through a 
lengthy process of capturing, transferring, printing and gluing images onto the labbooks, they saw value in doing so. As we pointed out, record keeping allowed them to troubleshoot, coordinate among collaborators. They also used lab-books as a reference tool to guide future research activities. It is also important to point out the lack of technology support. The use of smartphones was itself a technology use, but at several points we saw that researchers used hand-written labels, relied on physical scale bars for measurements and glued images on lab-books and annotated them. We believe that there is a huge scope for designing novel mobile technologies that can support biomedical engineering researchers' work practices. In the following we point to some interesting design considerations.

\subsection{Hybrid lab-books}

A quick look at the lab-books of our participants indicated how they combined a mix of digital and physical data into a paper-based book. They recorded digital information such as smartphone images, graphs and email prints and combined them with hand-written notes and drawings, post-its, and colorful bookmarks. This illustrates the versatile affordances of paper-based materials. On the one hand our research echoes the findings of Sellen and Harper's [27] work and highlights importance of technology non-use [1]. Sellen and Harper emphasized that the value of a physical artefact such as a paper is its materiality and affordances which allows for mobility, portability, sharability, which are not always easily substituted by a new digital paper technology. There is a clear value in sustaining the sanctity of a material artefact and a technology should build on these material qualities and not replace them. Similarly, the notion of technology non-use also proposes that in specific situations technology may not be the best solution.

On the other hand, our research highlights that technology was in fact used in combination with paper-based materials to produce images, graphs and email prints. Additionally, there were clear gaps where technology can actually improve parts of researchers' activities. Having an inbuilt measurement tool or a ruler on the screen of smartphone camera that can provide measures of specific physical samples; or digital labeling mechanisms through which individual samples can be identified; or being able to print images in a variety of formats and layouts - which can be later annotated if required; can be of great value and useful digital additions to the existing setup. These implications need to be experimented before any strong claims can be made.

HCI has witnessed some work on augmented lab-books [13, 15, 22, 25] and augmented workspaces [30] for biological researchers. Mackay et al.'s [15] participatory design approach showed a great potential. However, these research projects had a strong technology push. We believe the need for a hybrid lab-book still exists. A more balanced approach is required where users (biologists or biomedical scientists) can keep their existing practices of using a paper-based lab-book alive and smartphones can aid in minimizing the tedious tasks such as gluing printed images on paper labbook, labeling, and measuring. 


\subsection{Innovation through hardware and apps}

The field of biomedical engineering being visual and structural in nature provides an opportunity for designing innovative applications which utilizes the camera feature of smartphones. We clearly saw the need for developing a smartphone app to support much more refined ways to capturing and handling the microscopic images (Figure 1a). Currently a lot of appropriation and workarounds goes on where researchers would place their mobiles on the eye piece of microscopes and after some positioning they would capture images of their samples. This particular practice can be supported through very simple apps that can allow basic measurements, positioning, and printing incorporated in the smartphone itself. Advanced image processing and pattern recognition algorithms can allow for detecting and identifying specific objects from the samples or develop a much detailed morphological understanding of biological samples. Some progress is already visible where external hardware and sensors are used to aid specific features in the smartphone's capabilities. The example of CellScope [5] is a great one here. We believe that HCI researchers, utilizing user-centered perspectives, can lead the innovation in this domain.

\section{Conclusion}

In this paper, we showed our initial findings on the role smartphone images play in supporting scientific work. We do not claim this to be a comprehensive empirical account, however, our findings do point to some interesting tension between the use of physical and digital information in the field of biomedical engineering. Our findings highlight the different ways researchers incorporated the use of smartphone images in supporting their work. We believe that by having an account of the existing practices of using smartphone camera feature, HCI researchers will have a better handle toward designing innovative solutions to support biomedical scientists in their laboratory settings.

\section{Acknowledgement}

We thank all the participants of this study for their valuable time. We also thank the Science and Engineering Faculty, IFE and IHBI of QUT for funding this research.

\section{References}

1. Baumer, E. and Silberman, M.S. When the implication is not to design (technology). In Proceedings of the SIGCHI Conference on Human Factors in Computing Systems (CHI '11). ACM, New York, NY, USA, (2011), 2271-2274. 
2. Bourouis, A., Zerdazi, A., Feham, M. and Bouchachia, A. M-health: skin disease analysis system using smartphone's camera. Procedia Computer Science, 19, (2013), pp.11161120.

3. Braun, V. and Clarke, V. Using thematic analysis in psychology. Qualitative research in psychology 3, no. 2 (2006): 77-101.

4. Breslauer, D. N., Maamari, R. N., Switz, N. A., Lam, W. A., \& Fletcher, D. A. Mobile phone based clinical microscopy for global health applications. PloS one, 4(7), (2009). e6320.

5. CellScope ${ }^{\mathrm{TM}}$ 2016: https://www.cellscope.com/

6. Chhablani, J., Kaja, S., \& Shah, V. A. Smartphones in ophthalmology. Indian journal of ophthalmology, 60(2), (2012). 127.

7. Davis, E.A., Hovanesian, J.A., Katz, J.A., Kraff, M.C. and Trattler, W.B. Professional life and the smartphone. Cataract Refract Surg Today, (2010), pp.21-2.

8. Engestrom Y, Middleton D (1996) Cognition and Communication at Work, New York: Cambridge UP.

9. Gallegos, D., Long, K., Yu, H., Clark, P., Lin, Y., George, S., Nath, P. and Cunningham, B. Label-free biodetection using a smartphone. Lab on a Chip, 13(11), (2013) pp. 21242132.

10. Hutchins E (1995) Cognition in the Wild. Cambridge, MA: MIT Press.

11. Latour B (1986) Visualization and cognition: Thinking with eyes and hands, Knowledge and Society, 6: 1-40.

12. Latour B, Woolgar S (1986) Laboratory life: The construction of scientific facts. Princeton: Princeton University Press.

13. Klokmose, C. N., \& Zander, P. O. (2010). Rethinking laboratory notebooks. M. Lewkowicz et al. (eds.), Proceedings of COOP 2010, Computer Supported Cooperative Work.

14. Lynch M (2006) The production of scientific images: Vision and re-vision in the history, philosophy and sociology of science. In L. Pauwels (Ed.), Visual cultures of science: Rethinking representational practices in knowledge building and science communication (pp. 26-41). Hanover: Dartmouth College Press.

15. Wendy E. Mackay, Guillaume Pothier, Catherine Letondal, Kaare Bøegh, and Hans Erik Sørensen. 2002. The missing link: augmenting biology laboratory notebooks. In Proceedings of the 15th annual ACM symposium on User interface software and technology (UIST '02). ACM, New York, NY, USA, 41-50.

16. McNeill D (1995) Hand and Mind: What Gesture reveal about Thought. Chicago: University of Chicago Press.

17. Merleau-Ponty M (1962) Phenomenology of Perception. New York: Humanities Press.

18. Minger SL (2006) Regenerative medicine. Regenerative Medicine, Jan 2006, Vol. 1, No. 1 , Pages 1-2.

19. Monteiro M (2010) Reconfiguring evidence: interacting with digital objects in scientific practice. Computer Supported Cooperative Work (JCSCW), Springer 231-244.

20. Myers N (2008) Molecular embodiments and the body-work of modeling in protein crystallography. Social Studies of Science, 38(2), 163-199.

21. Norman D (1991) Cognitive artifacts. J.M. Carroll (Ed.), Designing interaction: Psychology at the human-computer interface, Cambridge University Press, New York. 
22. Oleksik, G., Milic-Frayling, N. and Jones, R. Study of electronic lab notebook design and practices that emerged in a collaborative scientific environment. In Proceedings of the 17th ACM conference on Computer supported cooperative work \& social computing. (2014), ACM, pp. 120-133.

23. Oncescu, V., Mancuso, M., Erickson. D. Cholesterol testing on a smartphone. Lab on a Chip. 14(4): 2014, 759-63.

24. Roy, R. C., \& Dadarya, S. Use of digital camera in clinical microbiological laboratory: an academic and diagnostic tool. International Journal of Biomedical and Advance Research, 3(5), (2012) 423-424.

25. m. c. schraefel, Gareth V. Hughes, Hugo R. Mills, Graham Smith, Terry R. Payne, and Jeremy Frey. 2004. Breaking the book: translating the chemistry lab book into a pervasive computing lab environment. In Proceedings of the SIGCHI Conference on Human Factors in Computing Systems (CHI '04). ACM, New York, NY, USA, 25-32.

26. Schmidt K, Wagner I (2002) Coordinative artefacts in architectural practice, in M. BlayFornarino et al. (eds.): Proceedings of the Fifth International Conference on the Design of Cooperative Systems, IOS Press, Amsterdam, 257-274.

27. Sellen A, Harper R (2002) The Myth of the Paperless Offices. MIT Press, MA.

28. Star SL (1985) Scientific Work and Uncertainty. Social Studies of Science, Vol. 15, No. 3 (Aug., 1985), Published by: Sage Publications, Ltd. pp. 391-427.

29. Suchman L (2000) Embodied practices of engineering work. Special issue of Mind, Culture and Activity, 7(1/2), 4-18.

30. Tabard, A., Hincapié-Ramos, J., Esbensen, M. and Bardram, J. The eLabBench: an interactive tabletop system for the biology laboratory. In Proceedings of the ACM International Conference on Interactive Tabletops and Surfaces, (2011), pp. 202-211. ACM. 